\title{
An Improved Gravitational Search Algorithm for Solving an Electromagnetic Design Problem
}

\author{
Talha Ali Khan and Sai Ho Ling \\ University of Technology Sydney, Ultimo, NSW, 2007, Australia
}

\begin{abstract}
Gravitational Search Algorithm (GSA) is a novel optimization technique that depends upon the law of motion and law of gravity of masses interaction between the agents. GSA has shown outstanding performance but has a drawback of having a slow exploitation process due to the fitness function influence on masses of the agents. As a result, after each iteration, the masses are getting heavier and restricts the movement. For that reason, masses cancel out the gravitational forces on each other and restricting them from quickly exploiting the optimum. To overcome this limitation, an improved GSA (IGSA) with a modified exploitation strategy is proposed in this paper. The primary aim of modification is to enhance the proficiency of the algorithm in terms of faster convergence and avoid premature convergence. An electromagnetic optimization problem is used to validate the performance of the presented method. Simulation results confirmed that the proposed method has outstanding results in solving the Loney's Solenoid design problem and solution stability is much better as compared to the Standard gravitational search algorithm and various other state-of-the-art techniques previously applied for solving this problem.
\end{abstract}

Index Terms-Gravitational Search Algorithm, Magnetic fields, Loney's solenoid problem, Optimization.

\section{INTRODUCTION}

The deterministic algorithms were very popular a few decades before, despite their incompetence to find global minima. Besides, the benchmark test functions required in this type of the numerical techniques must be not too "ill-conditioned" [1]. The most common example of an electromagnetic problem for such a rough test function surface is illustrated by Loney's solenoid benchmark problem that is appropriate for stochastic methods and will not be trapped from those local minima [2]. In the last decade, much more importance was given to the metaheuristic and stochastic methods.

There are many nature-based optimization algorithms used to solve different complex optimization problems. GSA is a novel technique that depends on the second law of motion and newton law of gravity. This algorithm emanates under the populationbased algorithm having agents of different masses [3] and has been applied to the number of the applications including power engineering [4-7], image processing [8-10], communication [11-13], controls [14-16], biology [17, 18], and computer science [19]. Every agent in GSA is simulated as a matter and problem search space as the universe where each agent subject to the gravitational force. The Einstein general theory of relativity defines as that the gravitational field is proven as a curvature of space-time[20]. As a result, many possibilities are available to explore the new operators and apply the theories of gravity. Many variants of the GSA have been developed in the last few years [21]. GSA has been, therefore, assessed as a population-based technique and a physics-based metaheuristic search algorithm too. Some of the operators defined from Newton's second law of motion are mass allotment, agents' movement, and calculation of the force experiencing by the agents. As the mass and distance between agents affect the force of gravity, the collaboration between the agents is taken place through the gravitational force. The amalgamation of all these factors that are: the relation between masses and the objective function, dependency on the distance, and combine the behavior of all the agents' gravitational force make the GSA algorithm unique. [22]. This algorithm works on the following mechanism. All the agents are attracted by the gravitational force. The force of gravity leads to the global mobility of all the agents in the direction of the agents having heavier masses. Agents with heavy masses have a higher force of gravity and travel slower than agents having lower masses. The exploitation phase is due to the agents having higher masses, and on the contrary, the exploration of the search area is due to the agents having lighter masses. At the starting of the searching process, the best solutions are distant from the optimal solution, therefore a large step size is required in the beginning i.e. exploration and later after some iterations as the agents (optimal solutions) are converged towards the optimum point, smaller step size is required for better exploitation of the search area. Therefore, in this paper in order to achieve faster convergence, stable solution, and to maintain a balance between exploitation and exploration a novel version of GSA is proposed.

\section{IMPROVED GRAVITATIONAL SEARCH ALGORITHM (IGSA)}

The mass of the agents evaluates the performance of the proposed method. In IGSA, the force of gravity attracts every agent, and it's accountable for the mobility of these agents towards the agents having heavier mass. Higher mass agents are considered as the best solution to the given problem. Since the physics laws inspire IGSA, every agent has four characteristics these are passive gravitational mass " $M_{p}$ ", inertial mass " $M_{i}$ ", active gravitational mass " $M_{a}$ ", and position.

Let's consider a system having " $N$ " agents; the position of the $i^{\text {th }}$ agent can be defined as:

$X_{i}=\left(X_{i}^{1}, X_{i}^{2}, \ldots \ldots \ldots \ldots X_{i}^{d}, X_{i}^{N}\right)$ for $i=(1,2, \ldots N)$

In the $d^{\text {th }}$ dimension, " $N$ " denotes as the dimension of the search area and " $x_{d}$ " represents the position of the $i^{\text {th }}$ object. The force exerting on mass " $i$ " from mass " $j$ " at time $t$ is represented as:

$F_{i j}^{d}(t)=G(t) \frac{M_{p i}(t) * M_{a j}(t)}{R_{i j}(t)+\varepsilon}\left(X_{j}^{d}(t)-X_{i}^{d}(t)\right)$

where $G(t)$ is a gravitational constant at time $t, R_{i j}(t)$ is the distance between two objects $i$ and $j, \varepsilon$ is a small constant, $M_{p i}$ is the passive gravitational mass related to object $i$, and $M_{a j}$ is an active gravitational mass of object $j$. 
Let's assume that in dimension $d$ the total force acted on object $i$ is a random weighted sum of all the components of the forces applied on the other agents then (2) can be rewritten as:

$F_{i}^{d}(t)=\sum_{j=1, j \neq \mathrm{i}}^{N} \operatorname{rand}_{j} F_{i j}^{d}(\mathrm{t})$

Define by the second law of motion at time $t$, the acceleration of the object $i$ in the direction $d$ can be written as:

$a_{i}^{d}(t)=\frac{F_{i}^{d}(t)}{M_{i i}(t)}$

For the $i^{\text {th }}$ agent, $M_{i i}$ is the inertial mass. The velocity of the agents is based on acceleration and their present velocity. The position and the velocity of every agent are updated by (5) and (6) as:

$X_{i}^{d}(t+1)=X_{i}^{d}+v_{i}^{d}(t+1)$

$V_{i}^{d}(t+1)=\operatorname{rand}_{i} * v_{i}^{d}(\mathrm{t})+a_{i}^{d}(t) *\left(1-\frac{t}{T}\right)^{\alpha}$

where rand $_{i}$ is a uniform random number within [0-1], $t$ is the current iteration, $T$ is the total number of iteration, which is the stopping criteria and $\alpha$ is an integer. Gravitational constant $G$ uses to monitor the searching accuracy, and it's decreasing over the iteration. In IGSA, the inertial mass and the gravitational masses are calculated by evaluating the fitness function. An agent with heavier mass is an efficient agent and indicates that agents with heavier masses have higher attractions and they move slowly. Suppose all the masses are equal so the inertial mass and the gravitational masses can be updated as follows:

$M_{a i}=M_{i i}=M_{p i}=M_{i} \quad i=1,2,3, \ldots \ldots \ldots N$

$m_{i}(t)=\frac{f i t_{i}(t)-X^{w}(t)}{X^{b}(t)-X^{w}(t)}$

$M_{i}(t)=\frac{m_{i}(t)}{\sum_{j=1}^{N} m_{j}(t)}$

At time $t, f i t_{i}(t)$ is the fitness value of the agent.

Worst $X^{w}(t)$ and Best $X^{b}(t)$ for a maximization problem are defined as:

$X^{b}(t)=\max _{j \varepsilon\{1, \ldots N\}} f i t_{j}(t)$

$X^{w}(t)=\min _{j \varepsilon\{1, \ldots N\}} f i t_{j}(t)$

For the minimization problem, (10) and (11) are defined as follows:

$X^{b}(t)=\min _{j \varepsilon\{1, \ldots N\} I} f i t_{j}(t)$

$X^{w}(t)=\max _{j \varepsilon\{1, \ldots N\}} f i t_{j}(t)$

To maintain a balance between exploration and exploitation in IGSA the number of agents is decreasing gradually until the agents with heavier masses only applying the force of gravity to the other agents in the search space are considered. This group of agents is known as Best agents " $K_{\text {best }}$ ". Therefore, the value of the $K_{\text {best }}$ is reducing slowly over the iterations till only one agent is applying force to the other agents. As a result, (3) is modified as follows:

$F_{i}^{d}(t)=\sum_{j \varepsilon K_{\text {best }}, j \neq \mathrm{i}} \operatorname{rand}_{j} F_{i j}^{d}(t)$
The pseudo-code of IGSA is as follows:

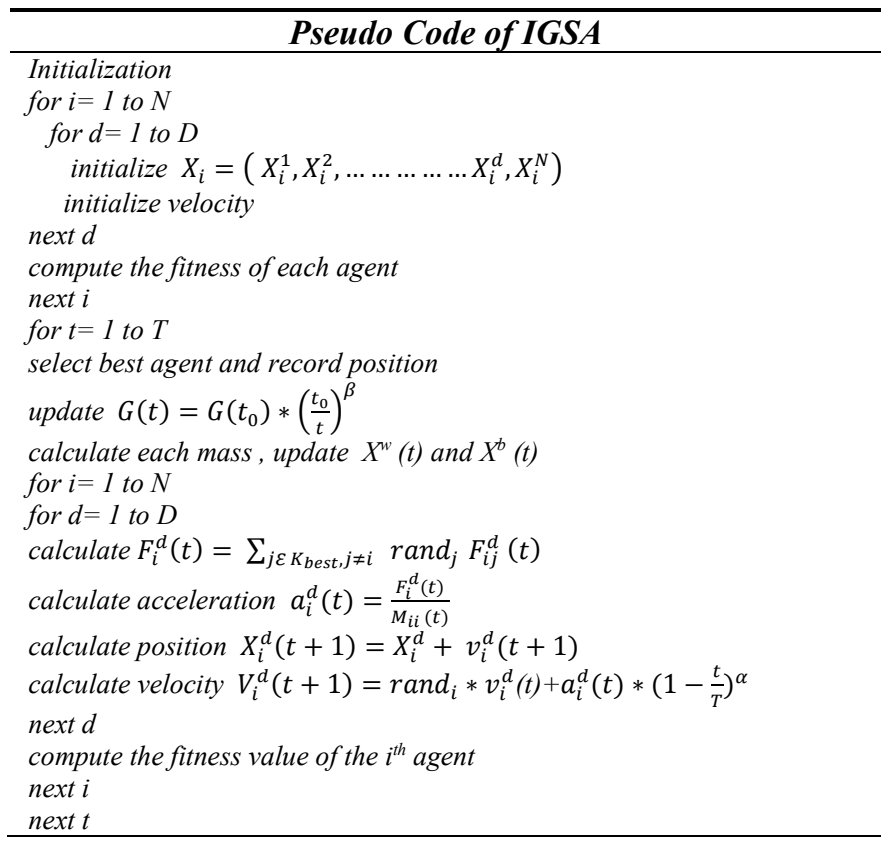

\section{LONEY'S SOLENOID BENCHMARK TEST PROBLEM}

Barba et al. [23] in 1995 introduced Loney's Solenoid problem. It is the most commonly used inverse optimization problem in the area of electromagnetics. It contains the main coil of fixed dimensions and two similar correcting coils located in such a way that the device is symmetric and the radial direction $\mathrm{z}=0$. The primary purpose of this problem is to find out the size and the position of two correcting coils so that these coils can generate a uniform magnetic flux distribution on the axis of the main solenoid.

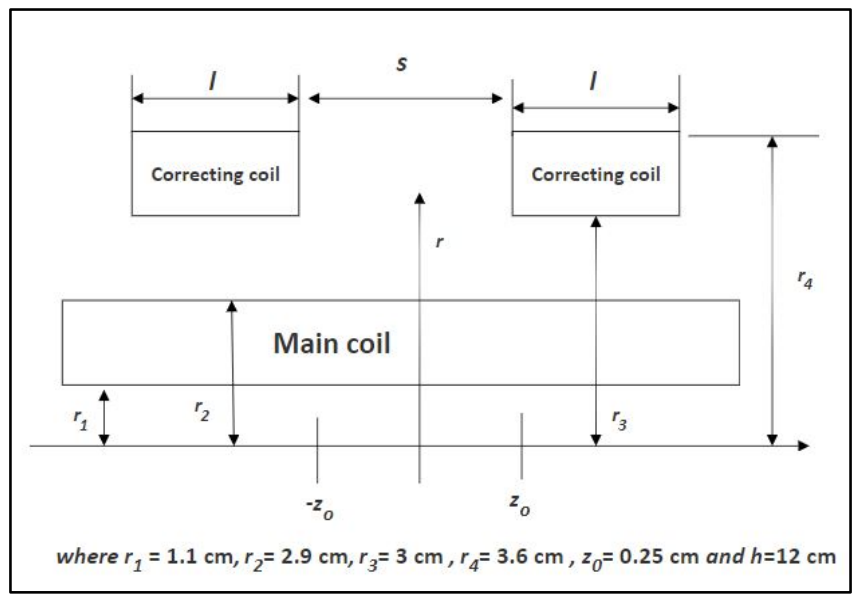

Figure 1 Axial cross section of Loney's solenoid.

Figure 1 shows the top half-plane of the axial cross-section of Loney's Solenoid. The objective of this paper is to determine the length of two coils and distance that they are separated from one another so that the magnetic flux density is constant. Assume that the constant current is flowing through the coil so that their current density is the same.

The problem to be mathematically solved is stated as: 
$\min F(s, l)$

The objective benchmark function is defined as [24]:

$F=\frac{B_{\max }-B_{\min }}{B_{0}}$

Where $B_{0}$ is the flux density at $\mathrm{z}=0, B_{\min }$ and $B_{\max }$ are the minima and maximum values of the magnetic flux density in the interval $\left(-z_{0}, z_{0}\right)$.

The magnetic field density obtained is the superposition of the main coil in which the correcting coils are discretized. Assuming the coordinate system is at the center of the solenoid the magnetic field density produced can be represented as

$B=\frac{\text { uin }}{2}\left(\cos \beta_{1}+\cos \beta_{2}\right)$

Where $u, i$ are the magnetic permeability, $n$ is the wind carrying the current and $\beta$ are the angles defined as:

$\cos \beta_{1}=\frac{\frac{H}{2}+Z}{\sqrt{R^{2}+\left(\frac{H}{2}+z\right)^{2}}}$

Similarly,

$\cos \beta_{2}=\frac{\frac{H}{2}-z}{\sqrt{R^{2}+\left(\frac{H}{2}-z\right)^{2}}}$

The term " $\frac{\text { uin }}{2}$ " is neglected in (17) as the coils have the same current density. The field produced is the superposition of the field produced by the main coil, and that does not contain the optimization parameters so the correcting coils fields can be defined:

$B(s, l)=B_{\text {main }}+B_{\text {correcting coils }}(s, l)$

To define the field of the main coil, let's assume that the main coil is discretized in $N$ solenoid of radius $r$. The field can be stated as:

$B_{\text {main }}=\sum_{k=1}^{N}\left(\cos \beta_{1}+\cos \beta_{2}\right)$

$r_{c 1}=r_{1}+\frac{r_{2}-r_{1}}{N-1}(k-1), \quad k=1,2, \ldots \ldots \ldots . . N$

Alternatively, if the correcting coils are discretized in the $N$ solenoid the field produced is the superposition of the field generated by the solenoids of length $(2 l+s)$ and radius $r_{c l}$ and solenoid of length $\mathrm{S}$ in which the current is flowing in the opposite direction. The field of the correcting coil is defined as:

$B_{\text {correcting coils }}(s, l)=\sum_{k=1}^{N}\left(\cos \beta_{1}+\cos \beta_{2}\right)-$

$\sum_{0 k=1}^{N}\left(\cos \beta_{1}+\cos \beta_{2}\right)=\sum_{k=1}^{N c}\left(B_{\text {correcting coils }-k}(S, L)\right.$

The first term in (22) is the field produced by the solenoids of the length $(2 l+s)$ and radius " $r_{c l}$ " and the second term is the field produced by the solenoid of length " $s$ " and radius " $r_{c 2}$ ".

Where $r_{c 2}=r_{3}+\frac{r_{4-} r_{3}}{N-1}(k-1), k=1,2, \ldots \ldots \ldots . . N$

By inserting the (18) and (19) in (23), the field that based on the optimization parameters are defined as:

$B_{\text {correcting coils }-k}(S, L)=\frac{\frac{2 L+S}{2}+z}{\left.\sqrt{\left(\left(r_{c 2}\right)^{2}\right.}+\left(\frac{2 L+S}{2}+z\right)^{2}\right)}+$
$\frac{\frac{2 L+S}{2}-z}{\left.\sqrt{\left(\left(r_{c 2}\right)^{2}\right.}+\left(\frac{2 L+S}{2}-z\right)^{2}\right)}-\frac{\frac{S}{2}+z}{\left.\sqrt{\left(\left(r_{c 2}\right)^{2}\right.}+\left(\frac{S}{2}+z\right)^{2}\right)}-\frac{\frac{S}{2}-z}{\left.\sqrt{\left(\left(r_{C 2}\right)^{2}\right.}+\left(\frac{S}{2}-z\right)^{2}\right)}$
The gradient of the objective function can be defined as

$\frac{\partial f}{\partial p}=\frac{1}{B_{O}^{2}}\left[\left(\frac{\partial B_{\max }}{\partial p}-\frac{\partial B_{\min }}{\partial p}\right) B_{O}-\left(B_{\max }-B_{\min }\right) \frac{\partial B_{O}}{\partial p}\right]$

where $p$ is either $S$ or $L, \frac{\partial B_{\max }}{\partial p}, \frac{\partial B_{\min }}{\partial p}$ are the derivatives assessed at the point where the field is maximum and minimum, and $\frac{\partial B_{O}}{\partial p}$ is the derivative estimated at the solenoid center. Calculating the derivative of the magnetic flux density $B$, only the terms coming from the correcting field matter.

$\frac{\partial B_{\text {correcting coils }-k}}{\partial S}(S, L)=\frac{\left(r_{C 2}\right)^{2}}{2\left(\sqrt{\left(r_{C 2}\right)^{2}+\left(\frac{2 L+S}{2}+z\right)^{2}}\right)^{3}}+$
$\frac{\left(r_{C 2}\right)^{2}}{2\left(\sqrt{\left(r_{C 2}\right)^{2}+\left(\frac{2 L+S}{2}-z\right)^{2}}\right)^{3}}-\frac{\left(r_{C 2}\right)^{2}}{2\left(\sqrt{\left(r_{C 2}\right)^{2}+\left(\frac{S}{2}+z\right)^{2}}\right)^{3}}-\frac{\left(r_{C 2}\right)^{2}}{\left.2\left(\sqrt{\left(r_{C 2}\right)^{2}+\left(\frac{S}{2}+z\right.}\right)^{2}\right)^{3}}$

$\frac{\partial B_{\text {correcting coils }-k}}{\partial L}=2 \frac{\partial B_{\text {correcting coils }-k}}{\partial S}$

(27) and (28) can be used for optimizing the objective function by calculating the gradient.

\section{RESULTS AND DISCUSSION}

To estimate the efficiency of the proposed method, an electromagnetic optimization problem is considered. Fig.2 shows that the Loney's Solenoid is a nonanalytic, illconditioned function presenting U-shaped, steep and narrow valley, and having many local minima. These minimum are located in various basins of interest in the domain of " $F$ ". Particularly, three basins have been recognized consisting to values of $F>4 \times 10^{-8}$ (High-Level Region: HL), $3 \times 10^{-8}<F<$ $4 \times 10^{-8}$ (Low-Level Region: LL) and $F<3 \times 10^{-8}$ (Global Minimum Region: GMR).

The specific U-shape of the function recommends proposing an optimization approach capable of exploring the most attractive basins intensely.

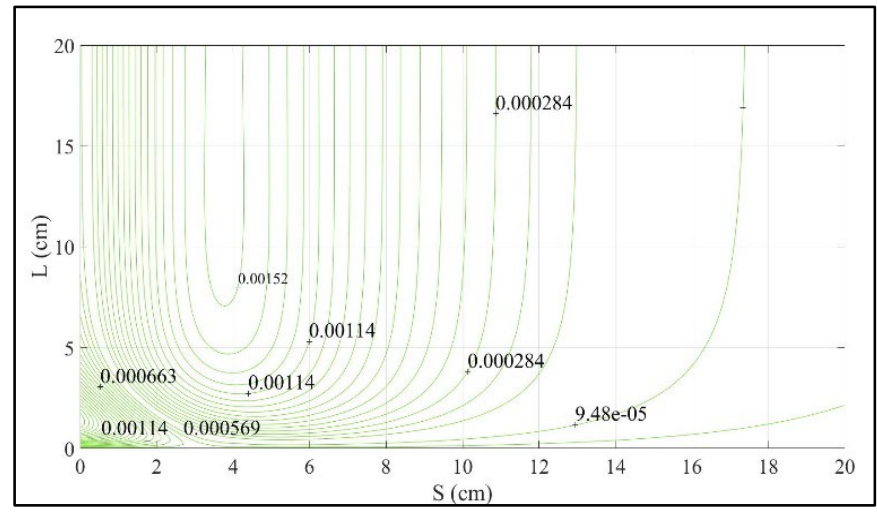

Figure $2 \mathrm{U}$-shaped basins of attraction of the objective function. 


\section{A. Comparison with state-of-the-art techniques applied to Loney's Solenoid Benchmark problem:}

IGSA is compared with the different state of the art algorithms that have been applied previously for solving this problem. These algorithms are:

\section{a) Gaussian Artificial Bee Colony Algorithm:}

Coelho et al. proposed a modified Artificial Bee Colony (IABC) approach. In the conventional $\mathrm{ABC}$ where uniform distribution is used for the candidate food positions, a new mechanism is utilized where random numbers extracted from a Gaussian distribution are used [25].

\section{b) Modified Blind Naked Mole-Rats (BNMR)} algorithm:

Reza et al. introduced an MBNMR algorithm in which by utilizing $\beta$-distribution instead of uniform distribution, the algorithm performance is improved than the classical BNMR method [26].

\section{c) Tribes Optimization Algorithm:}

Alotto et al. proposed a modified form of the particle swarm optimization algorithm that worked on the concept of the tribes. Loney's solenoid problem is used to test the efficiency of the algorithm[27].

\section{d) Quantum Behaved PSO:}

Different neighborhood strategies have been applied to the QPSO with random mean, the QPSO with Gaussian attractor and on the simple QPSO[28].

\section{e) Intelligent-particle swarm optimization (ISPO):}

Gabriela et al. proposed a novel ISPO algorithm for solving Loney's solenoid problem. They improved the algorithm by proposing concepts such as sharing group experiences, erased the unpleasant memories, introducing local landscape models depends upon virtual neighbors, and memetic repetition of successful behavior factors [29].

\section{f) Modified Stem cells algorithm:}

Mohammad proposed a modified version of the classical stem cells method by using $\beta$ distribution in place of uniform distribution to solve the optimization function [30].

g) Modified Ant Colony Optimization Algorithm $\left(A C O_{R}\right)$ :

Duca et al. introduced a modified ant colony method to study the influence of the ants on solving the two electromagnetic optimization problems [31].

\section{B. Statistical Analysis:}

To estimate the proficiency of the presented method, it is compared with the Standard Gravitational Search Algorithm (SGSA) and different state-of-the-art algorithms, as shown in table1.

The response of the term $\left(1-\frac{t}{T}\right)^{\alpha}$ with different values of " $\alpha$ " are shown in figure 3 . It is clear from fig. 3 if the values of the " $\alpha$ " are decreasing the response of the algorithm is steady and the agent is moving in the search space with the lower velocity. Also, for the lower values of " $\alpha$ ", for example, $\alpha=2$, the response of the algorithm is stable. As the term is multiplying with the acceleration of agents in (6), so if the acceleration is lower, the force of attraction is also lower and the mass of agent is increasing. In IGSA, the motivation is to enhance the exploitation of the proposed method, and by using this adaptive term, it makes the transit between exploration and exploitation steady. The step size is large at the beginning of the algorithm, and it decreases gradually over the iteration. Moreover, as the iteration increases the mass of the agents is also getting heavier and heavier, and the heaviest mass agent corresponds to the best solution. The idea of using this term is to make the process adaptive, so the heaviest mass moves slowly towards the optimal point at the end.

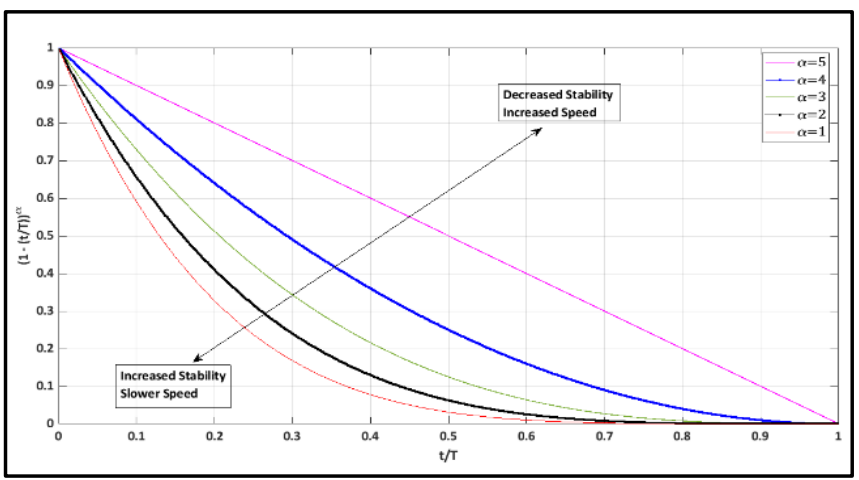

Figure 3 Response of " $\alpha$ " on different values on the term $\left(1-\frac{t}{T}\right)^{\alpha}$.

TABLE 1 Comparison of different algorithms for solving Loney's Solenoid Benchmark Function

\begin{tabular}{|c|c|c|c|c|}
\hline \multirow[b]{2}{*}{ Methods } & \multirow[b]{2}{*}{$S(\mathrm{~cm})$} & \multirow[b]{2}{*}{$L(\mathrm{~cm})$} & \multicolumn{2}{|c|}{$f(S, L)$} \\
\hline & & & Mean & $S D$ \\
\hline GABC $(\rho=0.1)$ & 14.5673 & 13.1474 & $2.2010 \mathrm{e}^{-08}$ & $0.570 \mathrm{e}^{-08}$ \\
\hline $\operatorname{GABC}(\rho=1)$ & 13.6104 & 4.0709 & $2.1073 \mathrm{e}^{-08}$ & $1 . .99 \mathrm{e}^{-08}$ \\
\hline M-BNMR & 12.00174 & 12.89026 & $1.09957 \mathrm{e}^{-08}$ & $0.278 \mathrm{e}^{-08}$ \\
\hline Tribes (PSO) & 14.56203 & 9.72521 & $2.0574 \mathrm{e}^{-08}$ & $5.23 \mathrm{e}^{-09}$ \\
\hline QPSO & - & - & $3.04 \mathrm{e}^{-08}$ & $3.47 \mathrm{e}^{-08}$ \\
\hline IPSO & 13.78 & 9.63 & $2.09846 \mathrm{e}^{-08}$ & $0.615 \mathrm{e}^{-08}$ \\
\hline MSCA & 13.47824 & 12.57024 & $2.00064 \mathrm{e}^{-08}$ & $0.4189 \mathrm{e}^{-08}$ \\
\hline $\mathrm{ACO}_{\mathrm{R}}$ & - & - & $2.22 \mathrm{e}^{-09}$ & $9.78 \mathrm{e}^{-09}$ \\
\hline SGSA & 14.8740 & 10.6631 & $3.5539 \mathrm{e}^{08}$ & $2.7721 \mathrm{e}^{-08}$ \\
\hline IGSA & 11.08923 & 3.89021 & $0.5619 \mathrm{e}^{-09}$ & $1.5391 \mathrm{e}^{-09}$ \\
\hline
\end{tabular}

Table 1 summarizes the comparison of the results of the IGSA with SGSA and various other state-of-the-art algorithms available in the literature. An analysis of table 1 illustrates that the IGSA outperforms the SGSA and other algorithms in terms of the mean and the standard deviation of the optimization function. IGSA method provides the best design results with $S=11.08923 \mathrm{~cm}$ and $L=3.89021 \mathrm{~cm}$.

\section{CONCLUSION}

IGSA is a new meta-heuristic algorithm depends upon the law of gravity. In this paper, an improved version of the standard GSA is presented by controlling the step size during the 
exploitation process to maintain a balance between exploitation and exploration.

IGSA is applied to an inverse ill-conditioned electromagnetic loney's solenoid problem to evaluate its proficiency. The results in terms of the mean and standard deviation values of this function validated the performance of the proposed algorithm and it shows better exploitative behavior in the final stages of the method than the other state-of-the-art techniques that have been already used to solve this problem.

\section{REFERENCES}

[1] L. d. S. Coelho and P. Alotto, "Gaussian artificial bee colony algorithm approach applied to Loney's solenoid benchmark problem," in Digests of the 2010 14th Biennial IEEE Conference on Electromagnetic Field Computation, 2010, pp. 1-1.

[2] E. Cogotti, A. Fanni, and F. Pilo, "Comparison of optimization techniques for Loney's solenoids design: An alternative Tabu Search algorithm," Magnetics, IEEE Transactions on, vol. 36, pp. 1153-1157, 08/01 2000.

[3] E. Rashedi, H. Nezamabadi-pour, and S. Saryazdi, "GSA: A Gravitational Search Algorithm," Information Sciences, vol. 179, no. 13, pp. 2232-2248, 2009/06/13/ 2009.

[4] B. Ji, X. Yuan, X. Li, Y. Huang, and W. Li, "Application of quantum-inspired binary gravitational search algorithm for thermal unit commitment with wind power integration," Energy Conversion and Management, vol. 87, pp. 589-598, 2014/11/01/ 2014.

[5] M. S. Jahan and N. Amjady, "Solution of large-scale security constrained optimal power flow by a new bi-level optimisation approach based on enhanced gravitational search algorithm," IET Generation, Transmission \& Distribution, vol. 7, no. 12, pp. 1481-1491, 2013.

[6] J. Radosavljević, M. Jevtić, N. Arsić, and D. Klimenta, "Optimal power flow for distribution networks using gravitational search algorithm," Electrical Engineering, vol. 96, no. 4, pp. 335-345, 2014/12/01 2014.

[7] S. Jiang, Z. Ji, and Y. Shen, "A novel hybrid particle swarm optimization and gravitational search algorithm for solving economic emission load dispatch problems with various practical constraints," International Journal of Electrical Power \& Energy Systems, vol. 55, pp. 628-644, 2014/02/01/ 2014.

[8] E. Rashedi and A. Zarezadeh, "Noise filtering in ultrasound images using Gravitational Search Algorithm," in 2014 Iranian Conference on Intelligent Systems (ICIS), 2014, pp. $1-4$.

[9] C. Gupta and S. Jain, "Multilevel fuzzy partition segmentation of satellite images using GSA," in 2014 International Conference on Signal Propagation and Computer Technology (ICSPCT 2014), 2014, pp. 173-178.

[10] W. Zhao, "Adaptive Image Enhancement Based on Gravitational Search Algorithm," Procedia Engineering, vol. 15, pp. 3288-3292, 2011/01/01/2011.

[11] A. Rahati Belabad, S. Sharifian, S. A. Motamedi, and N. Gholizadeh, "A novel model for digital predistortion based on a gravitational search algorithm for linearization of transmitters in LTE networks," Journal of Computational Electronics, journal article October 292019.

[12] P. Swain, S. K. Mohanty, and B. B. Mangaraj, "Linear Dipole Antenna Array design and optimization using Gravitational Search Algorithm," in 2016 2nd International Conference on Advances in Electrical, Electronics, Information, Communication and Bio-Informatics (AEEICB), 2016, pp. 514-518.
[13] P. A. Kumari and I. S. Prabha, "Optimum network selection in heterogeneous wireless environment using gravitational search algorithm," in 2015 International Conference on Signal Processing and Communication Engineering Systems, 2015, pp. 464-467.

[14] L. Wei and B. Ma, "Application of improved gravitational search algorithm in PID control for boiler drum water level," in 2017 29th Chinese Control And Decision Conference (CCDC), 2017, pp. 1852-1857.

[15] M. S. I. B. Aziz, S. W. Nawawi, S. Sudin, and N. A. Wahab, "Exploitation selection of alpha parameter in Gravitational Search Algorithm of PID controller for computational time analysis," in 2014 IEEE International Conference on Control System, Computing and Engineering (ICCSCE 2014), 2014, pp. 112-117.

[16] S. Duman, D. Maden, and U. Güvenç, "Determination of the PID controller parameters for speed and position control of DC motor using Gravitational Search Algorithm," in 2011 7th International Conference on Electrical and Electronics Engineering (ELECO), 2011, pp. I-225-I-229.

[17] J. Xiao and Z. Cheng, "DNA Sequences Optimization Based on Gravitational Search Algorithm for Reliable DNA Computing," in 2011 Sixth International Conference on BioInspired Computing: Theories and Applications, 2011, pp. 103-107.

[18] E. Zemali and A. Boukra, "EGSA: a new enhanced gravitational search algorithm to resolve multiple sequence alignment problem," International Journal of Intelligent Engineering Informatics, vol. 6, p. 204, 01/01 2018.

[19] M. Amoozegar and H. Nezamabadi-pour, "Software performance optimization based on constrained GSA," in The 16th CSI International Symposium on Artificial Intelligence and Signal Processing (AISP 2012), 2012, pp. 134-139.

[20] E. Rashedi, H. Nezamabadi-pour, and S. Saryazdi, "BGSA: binary gravitational search algorithm," Natural Computing, journal article vol. 9, no. 3, pp. 727-745, September 012010. [21] E. Rashedi, E. Rashedi, and H. Nezamabadi-pour, "A comprehensive survey on gravitational search algorithm," Swarm and Evolutionary Computation, vol. 41, pp. 141-158, 2018/08/01/2018.

[22] S. He, L. Zhu, L. Wang, L. Yu, and C. Yao, "A Modified Gravitational Search Algorithm for Function Optimization," IEEE Access, vol. 7, pp. 5984-5993, 2019.

[23] G. Di Barba, Savini, "Global optimization of Loney's solenoid: A benchmark problem," International Journal of Applied Electromagnetics and Mechanics (Int J Appl Electromagn Mech), vol. 6, no. 4, pp. 273-276 1995.

M.-I. Andrei, E. Caciulan, D. Dan, G. Ciuprina, and D. Ioan, Matlab Based Parallel Deterministic Optimization of the Loney's Solenoid. 2010.

[25] L. d. S. Coelho and P. Alotto, "Gaussian Artificial Bee Colony Algorithm Approach Applied to Loney's Solenoid Benchmark Problem," IEEE Transactions on Magnetics, vol. 47, no. 5, pp. 1326-1329, 2011.

[26] M. Taherdangkoo, "Modified BNMR algorithm applied to Loney's solenoid benchmark problem," International Journal of Applied Electromagnetics and Mechanics, vol. 46, 06/04 2014.

[27] L. D. S. Coelho and P. Alotto, "Tribes Optimization Algorithm Applied to the Loney's Solenoid," IEEE Transactions on Magnetics, vol. 45, no. 3, pp. 1526-1529, 2009.

[28] A. Duca, L.-C. Duca, G. Ciuprina, and D. Ioan, Neighborhood Strategies for QPSO Algorithms to Solve Benchmark Electromagnetic Problems. 2016, pp. 148-155. 
[29] G. Ciuprina, D. Ioan, and I. Munteanu, "Use of intelligentparticle swarm optimization in electromagnetics," IEEE Transactions on Magnetics, vol. 38, no. 2, pp. 1037-1040, 2002.

[30] M. Taherdangkoo, "Modified stem cells algorithm for Loney's solenoid benchmark problem," International Journal of Applied Electromagnetics and Mechanics, vol. 42, 03/01 2013.

[31] A. Duca, G. Ciuprina, S. Lup, and I. Hameed, $A C O R$ Algorithm's Efficiency for Electromagnetic Optimization Benchmark Problems. 2019, pp. 1-5. 